\title{
SÍNTESE DA ESTRUTURA DE PROCESSAMENTO DE UM PRODUTO À BASE DE CACAU: MAXIMIZAÇÃO DE COMPONENTES ANTIOXIDANTES
}

\author{
R. F. NASCIMENTO ${ }^{1}$, L. S. ARRIECHE ${ }^{1}$ \\ ${ }^{1}$ Universidade Federal do Espírito Santo, Departamento de Engenharias e Tecnologia \\ E-mail para contato: raulfn@gmail.com
}

\begin{abstract}
RESUMO - As etapas de processamento do cacau reduzem os compostos fenólicos, ou por difusão, ou por oxidação. Assim, o objetivo deste trabalho consistiu em sintetizar a estrutura de processamento do cacau, maximizando os teores dos compostos fenólicos, atribuindo ao chocolate melhores propriedades antioxidantes e sensoriais. Para isso, foram utilizados os Métodos Heurístico e Evolutivo. O processo de obtenção do chocolate foi estruturado na forma de uma árvore de estados, com seis subsistemas e 200 fluxogramas. Com a utilização de regras heurísticas desenvolveram-se algoritmos para identificação dos estados mais apropriados, que foram: fermentação com inibição química por $\mathrm{CuSO}_{4}$, secagem solar, torrefação em estufa sem circulação de ar com temperatura mais baixa $\left(80^{\circ} \mathrm{C}\right)$ e moagem em moinho de rolos. Assim, identificou-se o fluxograma base como sendo o de número 122. Pelo Método Evolutivo, identificaram-se doze fluxogramas vizinhos ao fluxograma 122, que possivelmente resolvem a função objetivo.
\end{abstract}

\section{INTRODUÇÃO}

Os produtos de cacau são ricos em alcaloides naturais, como a teobromina e a cafeína, além de antioxidantes de um grupo chamado de flavonóides, pertencentes à classe dos polifenóis; podendo melhorar a saúde cardiovascular, por apresentarem propriedades antioxidantes (RICHTER \& LANNES, 2007; AFOAKWA, 2010).

Diferentes etapas são aplicadas às amêndoas para a produção do líquor. Dentre estas, tem-se o corte do fruto e a fermentação, onde as amêndoas são dispostas em barcaças e, durante sete dias, as leveduras $S$. cerevisae e $C$. krusei atuam desenvolvendo precursores do sabor característicos do chocolate (OETTERER et al., 2006). Esta etapa representa cerca de $70 \%$ na redução dos compostos fenólicos. Perdas consideráveis ainda podem ocorrer em etapas posteriores à fermentação, principalmente na torrefação, onde o tempo de torra relaciona-se inversamente proporcional aos teores de polifenóis totais (EFRAIM et al., 2010).

Após a fermentação, o desenvolvimento de sabor a partir de precursores do cacau continua durante a secagem, com o desenvolvimento de cor marrom característica (AFOAKWA, 2010). A secagem é responsável pela diminuição da umidade das amêndoas para uma faixa de 6 a $8 \%$ (b.u.). A terceira etapa, de torrefação, consiste em uma nova secagem para que a umidade chegue a cerca de $2 \%$ (b.u.). Ao final, as amêndoas de cacau 
estão prontas para a moagem, onde é feita a redução granulométrica das partículas sólidas até a estrutura mássica atingir um estado fluido de alta viscosidade (LOPES, 2000).

Perante a diversidade de tecnologias para a transformação do cacau em chocolate, configurações distintas podem ser adotadas para a estrutura de processamento. A determinação da rota e dos equipamentos mais adequados ao processo é feita por meio de regras heurísticas, que consistem numa reunião de regras práticas, com base na fundamentação teórica e na experiência acumulada. $\mathrm{O}$ aprimoramento das regras heurísticas pode ser feito por meio do método evolutivo, que introduz uma estratégia que questiona sua validade, alcançando a solução mais próxima da ótima (PERLINGEIRO, 2005).

Com base no exposto, o objetivo deste trabalho consiste em sintetizar a estrutura de processamento de um produto à base de cacau, de modo que os teores dos compostos fenólicos sejam maximizados no produto final, atribuindo ao chocolate melhores propriedades antioxidantes e qualidade sensorial.

\section{METODOLOGIA}

\subsection{Subsistemas do Processamento}

Separação de Polpa: Neste procedimento foram propostas quatro rotas, fermentação tradicional (F1), fermentação com inibição por $\mathrm{NaHSO}_{3}$ (F2), fermentação com inibição por $\mathrm{CuSO}_{4}(\mathrm{~F} 3)$ e maceração em água (F4) (EFRAIM, 2004; OETTERER et al., 2006).

Secagem: Foram avaliadas cinco rotas, secagem em estufa (S1), secagem convectiva (S2), secagem solar (S3), e secagem solar combinada com secagem em estufa (S4) e secagem solar combinada com secagem convectiva (S5), segundo Guehi et al. (2010).

Torrefação: Foi realizada em duas abordagens, através de estufa sem circulação de ar, com temperaturas de $80^{\circ} \mathrm{C}$ (T1) e $150^{\circ} \mathrm{C}$ (T2) e em estufa com circulação de ar, com temperaturas de $80^{\circ} \mathrm{C}(\mathrm{T} 3)$ e $150^{\circ} \mathrm{C}(\mathrm{T} 4)$. Também foi proposta a eliminação desta etapa (T5).

Moagem: Foram utilizadas duas abordagens, a moagem em moinho de bolas (M1) e a moagem em moinho de rolos de granito (M2).

\subsection{Determinação da Árvore de Estados, do Fluxograma Base e dos Vizinhos Estruturais}

A partir da enumeração de equipamentos para cada subproblema, determinaram-se os fluxogramas plausíveis para a obtenção do produto final. Estes, então, foram avaliados quanto ao seu desempenho. Pela aplicação das regras heurísticas, determinou-se o fluxograma base. De posse do fluxograma base, foram determinados os fluxogramas vizinhos pelo método evolutivo. Os vizinhos diferem-se entre si em apenas um dos subsistemas. 


\section{RESULTADOS E DISCUSSÕES}

\section{1. Árvore de Estados}

O problema de síntese está baseado na elaboração da árvore de estados, em que cada ramo é uma rota possível do processamento e não reflete, ainda, a solução do problema. Para as etapas de separação da polpa, secagem, torrefação e moagem foram verificadas outras possibilidades de processamento. Desta forma, com a combinação dos subsistemas avaliados, houve a geração de 200 rotas possíveis para o processamento, conforme a Figura 1.

Figura 1 - Árvore de estados do processamento do cacau para produção de chocolate.

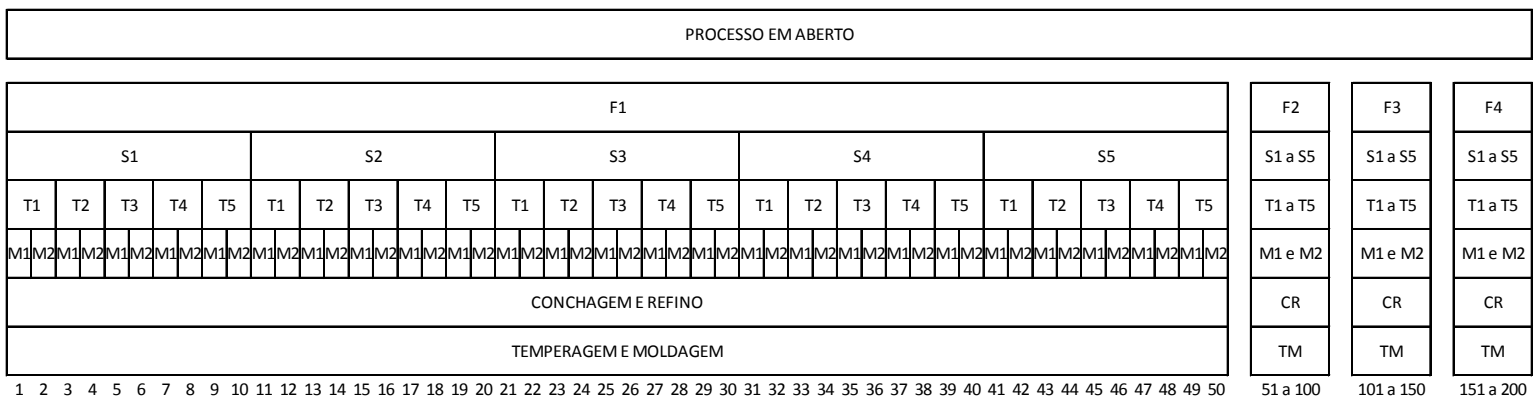

\subsection{Regras Heurísticas e Fluxograma Base}

A primeira etapa do processamento do cacau corresponde à perda da polpa (OETTERER et al., 2006; EFRAIM et al., 2006). As regras heurísticas foram reunidas na forma de um algoritmo, representado na Figura 2.

Figura 2 - Algoritmo para a escolha do tipo de separação de polpa.

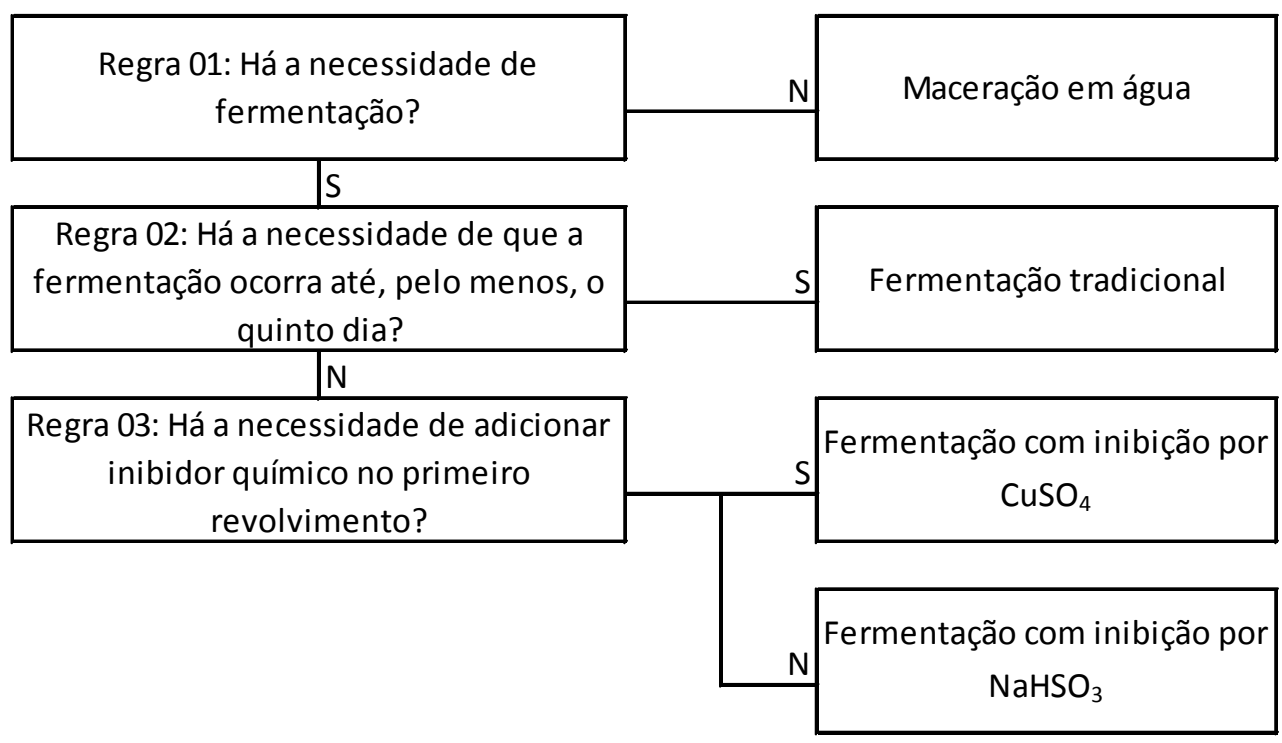


Caso a polpa seja retirada sem processo fermentativo, espera-se que os compostos fenólicos não sejam degradados, já que não haverá reações bioquímicas. Entretanto, a qualidade sensorial fica prejudicada. De modo inverso, na fermentação tradicional, a redução dos compostos fenólicos das amêndoas chega a $70 \%$ e sabor e aroma são bem desenvolvidos. Portanto, nesta etapa do algoritmo, um objetivo é muito bem atendido e o outro não. $\mathrm{Na}$ hipótese de que haja necessidade de inibição química no primeiro revolvimento, este é feito com $\mathrm{CuSO}_{4}$, e há perda de 37,30\% dos compostos fenólicos e aceitabilidade sensorial maior ou igual ao produto tradicional. Pela hipótese negativa, a perda de compostos fenólicos foi de $47,20 \%$ e a resposta sensorial, menor em comparação ao produto tradicional (EFRAIM, 2004). A melhor rota estabelecida pela Figura 2 é fermentação com inibição por $\mathrm{CuSO}_{4}$ (F3).

$\mathrm{Na}$ secagem, um tratamento térmico é realizado a fim de reduzir a umidade das amêndoas fermentadas. Usa-se geralmente exposição ao sol ou técnicas que utilizam ar aquecido, dependendo do tipo de cacau, quantidade de sementes e condições climáticas. Além de técnicas isoladas, pode-se haver a combinação de secagem natural com artificial. As regras heurísticas para a escolha do tipo de secagem estão representadas na Figura 3.

Figura 3 - Algoritmo para a escolha do tipo de secagem.

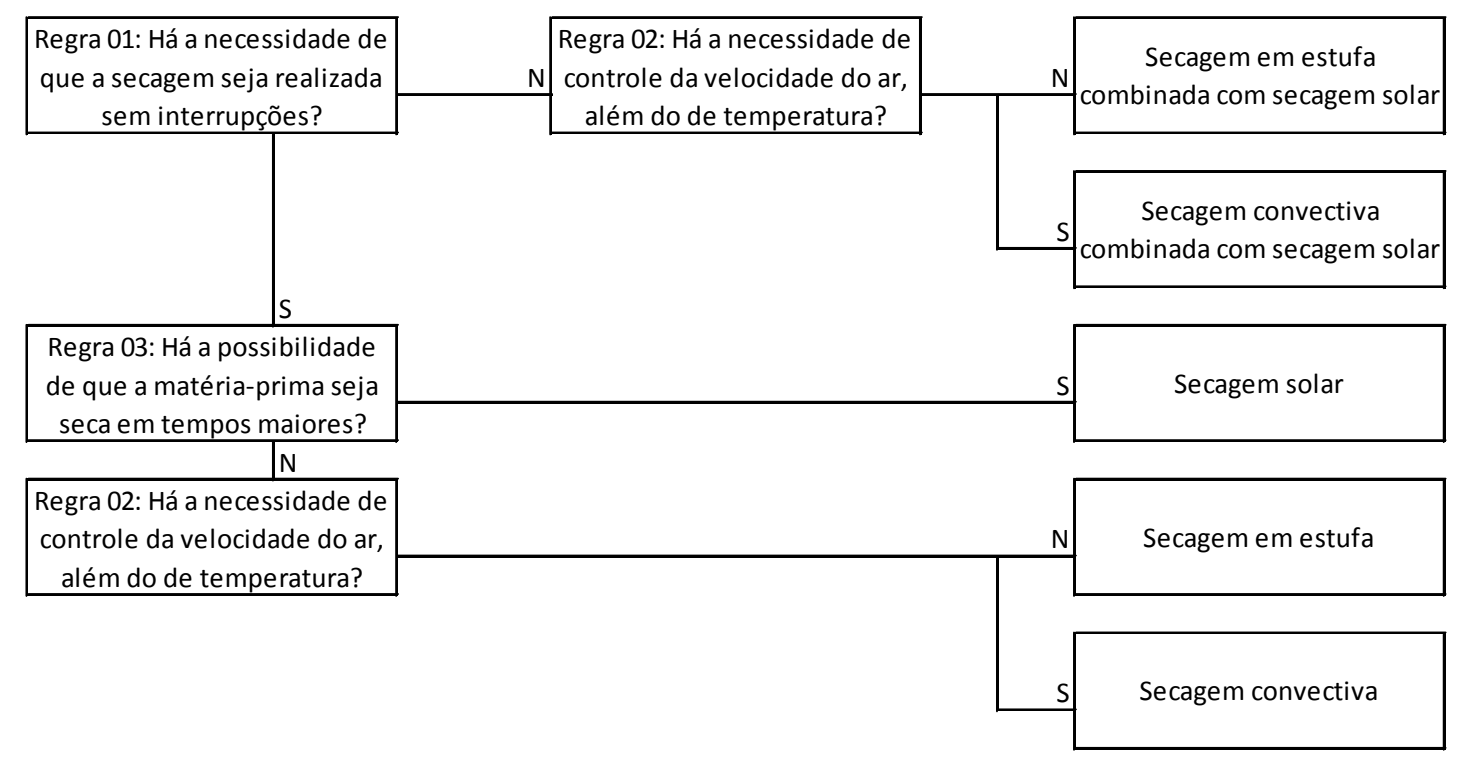

Optar por realizar técnicas isoladas ou a combinação não gera prejuízo na qualidade sensorial nem na quantidade de compostos fenólicos. Com temperaturas mais elevadas e com maiores velocidades de ar aquecido, a secagem ocorre de forma mais rápida. Mas, secagem realizada em tempos muito curtos afeta a qualidade das sementes, faz a manteiga de cacau migrar para a superfície e interfere no desenvolvimento do sabor. Assim, a melhor rota para este subsistema é a secagem solar (S3).

Após a secagem, a umidade das amêndoas está em aproximadamente 7\%, mas esta deve ainda ser diminuída até atingir por volta de $2 \%$. Entretanto, esta etapa não garante que os compostos fenólicos ainda presentes permaneçam nas amêndoas, já que esta também é uma etapa de tratamento térmico. A aplicação da torrefação proporciona ao chocolate 
características sensoriais, redução de ácidos voláteis e inativação de enzimas que degradam a manteiga de cacau. As regras para esta etapa encontram-se na Figura 4.

Figura 4 - Algoritmo para a escolha do tipo de torrefação.

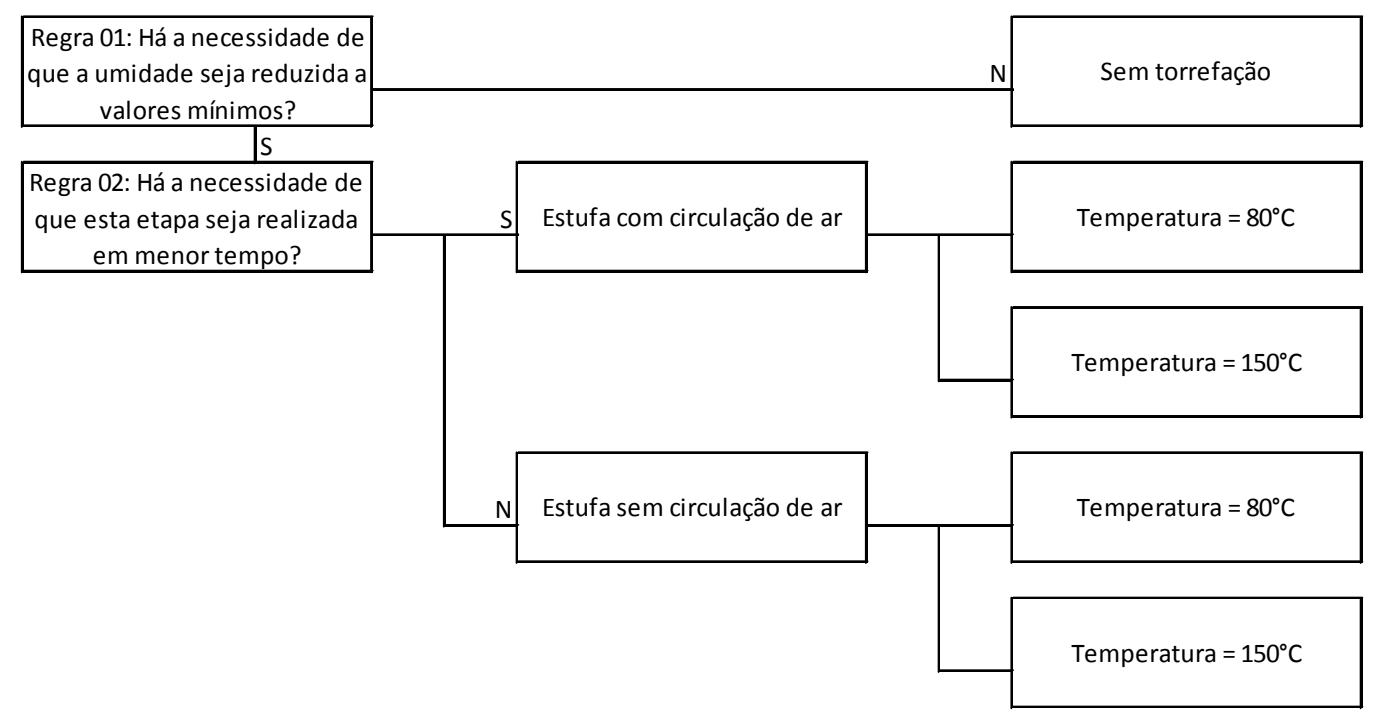

Supondo-se a escolha da rota 'Sem torrefação', acredita-se que a quantidade de compostos fenólicos ao final desse subsistema será igual ao final da secagem. Todavia, a qualidade sensorial fica comprometida, uma vez que ácidos permanecem nas amêndoas. Avaliando tempo e temperatura, maiores tempos de operação e temperaturas inferiores provocam maiores perdas dos compostos voláteis; e temperaturas mais elevadas provocam a destruição de compostos importantes, verificando sua desvantagem. A rota selecionada foi sem circulação de ar com temperatura de $80^{\circ} \mathrm{C}$ (T1).

$\mathrm{Na}$ moagem foram avaliadas duas alternativas, moinho de rolos e moinho de bolas. $\mathrm{O}$ primeiro é formado por rolos que giram com velocidades diferentes e em direções opostas, sendo mais indicado para a moagem das amêndoas inteiras ou parcialmente quebradas, de tamanho de partícula próximo a $5 \mathrm{~mm}$. Já o moinho de bolas é indicado para moagens como a que acontece no refino do chocolate. Desta forma, a escolha foi o moinho de rolos (M2).

De posse do que foi apresentado, determinou-se o fluxograma base pela junção e ordenação das escolhas feitas pelas regras heurísticas aplicadas a cada um dos subsistemas. $\mathrm{O}$ fluxograma base do processo é o correspondente a ordenação F3-S3-T1-M2-CR-TM, ou aquele representado na Figura 1 pelo número 122.

\subsection{Vizinhos Estruturais}

A identificação dos vizinhos estruturais faz parte da técnica de análise do Método Evolutivo. Como apresentado anteriormente, o fluxograma base foi o fluxograma de número 122 ou a sequência F3-S3-T1-M2-CR-TM, na Figura 1. Ao total foram determinados doze vizinhos estruturais do fluxograma 122, que são em ordem crescente: $22,72,102,112,121$, $124,126,128,130,132,142$ e 172. 


\section{CONCLUSÕES}

A metodologia utilizada se mostrou adequada, mediante a aplicação da representação por meio de árvore de estados para o problema de síntese de processos. A elaboração da árvore de estados relacionou-se ao processamento do cacau em chocolate e a sequência estudada fez com que fossem gerados 200 fluxogramas diferentes para um mesmo processo.

Quando se analisou cada subsistema individualmente, com foco na função objetivo de maximização de componentes antioxidantes e da qualidade sensorial, desenvolveram-se regras heurísticas, com fundamentos em pesquisa documental. Os principais resultados para cada subsistema são: Subsistema de Separação de Polpa: F3 - fermentação com inibição química por $\mathrm{CuSO}_{4}$; Subsistema de Secagem: S3 - secagem solar; Subsistema de Torrefação: $\mathrm{T} 1$ - torrefação em estufa sem circulação de ar com temperatura mais baixa $\left(80{ }^{\circ} \mathrm{C}\right)$; Subsistema de Moagem: M2 - moinho de rolos.

O fluxograma base foi identificado pela ordenação sequencial dos resultados alcançados pelos algoritmos. Dentre os 200 fluxogramas inicialmente obtidos, somente um foi identificado como mais promissor na resolução do problema principal deste trabalho, o fluxograma 122.

\section{REFERÊNCIAS}

AFOAKWA, E. O. Chocolate science and technology. Chichester: Wiley-Blackwell, 2010.

EFRAIM, P. Estudo para minimizar as perdas de flavonoides durante a fermentação de sementes de cacau para a produção de chocolate. Faculdade de Engenharia de Alimentos, UNICAMP, Campinas, 2004.

EFRAIM, P.; PEZOA-GARCÍA, N. H.; JARDIM, D. C. P.; NISHIKAWA, A.; HADDAD, R.; EBERLIN, M. N. Influência da fermentação e secagem de amêndoas de cacau no teor de compostos fenólicos e na aceitação sensorial. Ciênc. Tecnol. Aliment., Campinas, v. 30, p. 142-150, 2010.

GUEHI, T. S.; ZAHOULI, I. B.; BAN-KOFFI, L.; FAE, M. A.; NEMLIN, J. G. Performance of different drying methods and their effects on the chemical quality attributes of raw cocoa material. International Journal of Food Science and Technology, v. 45, p. 15641571, 2010.

LOPES, A. S. Estudo químico e nutricional de amêndoas de cacau (Theobroma cacao L.) e cupuaçu (Theobroma grandiflorum Schum) em função do processamento. Faculdade de Engenharia de Alimentos, UNICAMP, Campinas, 2000.

OETTERER, M.; REGITANO-D'ACRE, M. A. B.; SPOTO, M. H. F. Fundamentos de ciência e tecnologia de alimentos. Barueri-SP: Manole, 2006.

PERLINGEIRO, C. A. G. Engenharia de processos: análise, simulação, otimização e síntese de processos químicos. Editora Blucher, 2005.

RICHTER, M.; LANNES, S. C. S. Ingredientes usados na indústria de chocolates. Rev. Bras. Ciênc. Farm., São Paulo, v. 43, n. 3, set. 2007. 